\title{
Study Site Personnel Role Code
}

National Cancer Institute

\section{Source}

National Cancer Institute. Study Site Personnel Role Code. NCI Thesaurus. Code C94149.

The coded value specifying a type of responsibility of the study site personnel. 